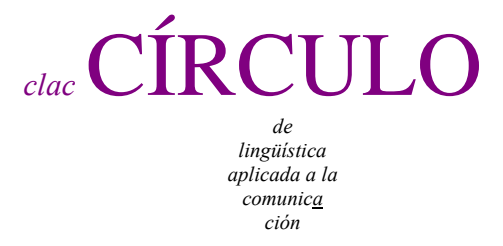

$54 / 2013$

\title{
EL VALOR ASPECTUAL DE LOS VERBOS REVERSATIVOS: \\ CLAVES PRAGMÁTICAS PARA UN PROCESO DE VERBALIZACIÓN \\ Susana Rodríguez Rosique
}

Universidad de Alicante

susana rodriguez en ua es

\section{Resumen}

El valor reversativo es un tipo de oposición entre verbos que puede manifestarse léxica o morfológicamente. En el caso del español, el reversativo es uno de los valores más productivos del prefijo des- cuando se une a bases verbales para formar otros verbos mediante derivación. Tradicionalmente se ha asumido que este valor se desencadena cuando el prefijo se une a bases télicas, por lo que, en consecuencia, se deduce que el derivado es también télico. El propósito de este artículo es explicar el comportamiento de los verbos reversativos debido a su carácter marcado, en el sentido de que aluden a un estado anterior. El tipo de oposición entre el estado resultante de la base y el estado resultante del derivado se refleja en la imagen verbal del derivado. Asimismo, se mostrará cómo, en muchas ocasiones, un principio de refuerzo pragmático contribuye a la telicidad y ayuda a perfilar la imagen aspectual del verbo reversativo. Desde una perspectiva más general, este trabajo plantea la importancia de tener en cuenta los principios pragmáticos en el análisis de las categorías gramaticales.

Palabras clave: oposición, verbos reversativos, aspecto, telicidad, refuerzo pragmático.

Rodríguez Rosique, Susana. 2013.

El valor aspectual de los verbos reversativos: Claves para un proceso de verbalización Círculo de Lingüística Aplicada a la Comunicación 54, 99-129.

http://www.ucm.es/info/circulo/no54/rosique.pdf

DOI http://dx.doi.org/10.5209/rev_CLAC.2013.v54.42374

(C) 2013 Susana Rodríguez Rosique

Círculo de Lingüística Aplicada a la Comunicación (clac)

Universidad Complutense de Madrid. ISSN 1576-4737. http://www.ucm.es/info/circulo 


\begin{abstract}
The reversative value is a kind of opposition between verbs which may manifest lexical or morphologically. In the case of Spanish, the reversative is one of the most productive values of the prefix des- when it joins to verbal bases in order to create new verbs. Traditionally it has been assumed that this value is triggered when the prefix is adhered to telic bases, and as consequence the reversative is also conceived as telic. The aim of this paper is to explain the behavior of reversative verbs in terms of their "marked" nature, in the sense that they allude to a previous state. The kind of opposition between the base verb and the derived verb is in a way reflected in the verbal image of the reversative. More generally, this paper highlights the importance of pragmatic principles in the analysis of grammatical categories.
\end{abstract}

Key words: opposition, reversative verbs, aspect, telicity, pragmatic reinforcement.

Índice

1. Introducción: Revisitando el valor reversativo 101

2. La difícil caracterización de los verbos reversativos: Un valor “líquido” 103

3. Escalaridad y aspecto: visiones y revisiones 107

4. Un tipo de oposición “muy verbal” 114

4.1. Reversativos que reflejan una relación de oposición complementaria entre el estado resultante de la base y el estado resultante del derivado. 114

4.2. Reversativos que reflejan una relación de oposición antonímica ${ }_{1}$ entre el estado resultante de la base y el estado resultante del derivado 119

4.3. Reversativos que reflejan una relación de oposición antonímica 2 entre el estado inicial y el estado resultante del derivado 121

5. Conclusión 123

Bibliografía 124 


\section{Introducción: Revisitando el valor reversativo}

El valor reversativo es un tipo de oposición, o, en palabras de Lyons (1977), un contraste entre dos términos; de manera más específica ${ }^{*}$, se trata de una relación de oposición entre verbos, donde el significado de uno de ellos invierte el significado del otro $^{1}$. Este tipo de relación contrastiva puede manifestarse léxicamente, como demuestra la vinculación que existe entre entrar / salir o entre subir / bajar; o morfológicamente, como sucede en parejas del tipo hacer / deshacer. En este sentido, el reversativo es uno de los valores más frecuentes del prefijo des- cuando se une a bases verbales para formar otros verbos (Brea 1994; Vañó-Cerdá 1990; Serrano Dolader 1995, 1999, 2011; Montero Curiel 1999; Valera y Martín García 1999; Martín García 2007; RAE 2009).

Los verbos reversativos morfológicos pueden considerarse formas marcadas, en términos de Givón (1978). En efecto, las formas vinculadas de alguna manera con la negación remiten a una información previa, o “que está en el aire” (Givón 1979; Horn [1989] 2001). Así, si un profesor se encuentra a un colega al que hace años que no veía, $\mathrm{y}$, al preguntarle cómo le va la vida, este le contesta con un enunciado afirmativo, como mi mujer está embarazada, el compañero puede alegrarse por la noticia, felicitarlo, etc., pues considerará que la información que le ha proporcionado su interlocutor es

\footnotetext{
* Este trabajo se enmarca en el seno del Proyecto Emergente "El aspecto verbal en español: Aplicaciones sintácticas, semánticas y pragmáticas en casos de verbalización” (GRE11-17), concedido por la Universidad de Alicante, y del Proyecto “Clases verbales y alternancias en la estructura argumental” (FFI2010-19946), concedido por el Ministerio de Ciencia e Innovación. Quisiera dar las gracias a Olga Batiukova, a Beth Levin y a los miembros del grupo LeXis de la Universidad de Alicante, por sus comentarios, críticas y referencias. Cualquier error de interpretación u omisión es exclusivamente responsabilidad mía.

${ }^{1}$ Para Cruse (1986), en la relación reversativa cada miembro de la pareja se mueve en direcciones opuestas; aunque la dirección no ha de entenderse únicamente en sentido físico, sino que puede proyectarse también sobre el cambio de estado -asumiendo la hipótesis localista de Lyons (1977)-. En concreto, el autor señala que son pocos los casos de reversativos que pueden explicarse en sentido puramente espacial, como sucede con subir / bajar; lo normal es que se entiendan en sentido abstracto.
} 
suficientemente informativa. Sin embargo, si, en la misma situación, el profesor responde mi mujer no está embarazada, la intervención podría considerarse cuando menos extraña, pues los enunciados negativos son estructuras marcadas con respecto a sus contrapartidas afirmativas. De igual forma, los reversativos morfológicos son formas marcadas, pues siempre remiten a un estado anterior con respecto al cual el estado resultante del verbo derivado mantiene una relación de oposición. En concreto, deshacer desemboca en un estado resultante (deshecho) que mantiene una relación de oposición con respecto al estado resultante de la base (hecho). Esta relación de oposición se refleja en la imagen aspectual del verbo, o en la disposición del evento.

El objetivo de este trabajo es proponer que existen diversos tipos de reversativos morfológicos, creados a partir del prefijo des-, en función del tipo de oposición que se establece entre el estado resultante del predicado base y el estado resultante del predicado derivado. Asimismo, se demostrará cómo determinadas inferencias sistemáticas ayudan a perfilar la imagen aspectual que tenemos de los reversativos; de manera más específica, se explicará cómo en muchas ocasiones la telicidad de los verbos reversativos no es más que una implicatura conversacional generalizada, desencadenada por un principio conversacional basado en el refuerzo pragmático de formas débiles.

Para llevar a cabo esta propuesta, el artículo se ha estructurado de la siguiente manera. En el apartado 2 se presentan los verbos reversativos y se explica cómo los dos rasgos que se han utilizado para caracterizarlos resultan controvertidos. En el apartado 3 se plantea un acercamiento a la categoría del aspecto, con especial atención a la perspectiva escalar. El apartado 4 aborda el análisis de distintos tipos de reversativos en función del tipo de oposición que se establece entre los estados resultantes de base y derivado. Finalmente, el apartado 5 cierra el trabajo con las conclusiones alcanzadas. 
2. La difícil caracterización de los verbos reversativos: Un valor “líquido”

Uno de los primeros autores en llamar la atención sobre los verbos reversativos fue Marchand ([1973] 1974), quien lleva a cabo un estudio contrastivo de los valores ablativo, privativo y reversativo que pueden desencadenar distintos prefijos negativos en lenguas como el inglés, el alemán o el francés cuando participan en procesos de verbalización. Estos tres valores pueden desencadenarse también en español mediante el prefijo des- cuando participa en la formación de verbos, como han puesto de manifiesto distintos autores en el ámbito de la morfología (Brea 1994; Vañó-Cerdá 1990; Serrano Dolader 1995, 1999, 2011; Montero Curiel 1999; Valera y Martín García 1999; Martín García 2007; RAE 2009):

-El valor ablativo está relacionado con el sentido de 'distancia', ‘alejamiento’ o, simplemente, 'movimiento' desde una base. Se observa en verbos como des-terra-r ('alejar de la tierra') o des-carril-ar ('alejar, salir del carril'). A partir de diversas conexiones semánticas, el valor de alejamiento desde un punto puede incluir también la idea de orientación ('des-peña-r', en el sentido de 'alejarse, caer desde una peña'), destacar el instrumento con el que se lleva a cabo el movimiento (des-pinza-r), o insistir en el resultado (des-troz-ar).

-El valor privativo está vinculado con la privación de alguna entidad poseída, sea de forma alienable (des-pioj-ar, con el sentido de 'quitar, o privar de piojos') o inalienable (des-cabeza-r, como 'quitar la cabeza') ${ }^{2}$. Igualmente, a partir de este valor se podrían explicar algunas extensiones semánticas que tienen que ver con 'golpe fuerte' o ‘dolor' en alguna parte del cuerpo (des-riñon-ar).

-El valor reversativo es el que codifica un verbo que invierte el significado de su base, como sucede en des-bloquear o des-calzar.

\footnotetext{
${ }^{2}$ Nótese que muchos de los verbos ablativos, como desterrar, descarrilar o despeñar son verbos de localización, mientras que los verbos claramente privativos, como despiojar o descabezar, son verbos locatum (Clark y Clark 1979). En términos de amalgama semántica, o según los patrones de lexicalización de Talmy (2000), los primeros incorporan la base, mientras que los segundos incorporan la figura (Cf. Cifuentes Honrubia 1999; 2006).
} 
Para Marchand, lo que tienen en común los tres valores (ablativo, privativo y reversativo) es la inversión de un estado previo; o, como él afirma, "the undoing of a previous state”. Por su parte, lo que diferencia a los reversativos de los otros dos tipos de verbos es la disponibilidad de una acción previa. Por ello, mientras que los valores ablativo y privativo suelen vincularse con casos de parasíntesis verbal -o aplicación simultánea de un prefijo y un sufijo para crear un nuevo verbo (Serrano Dolader 1995, 1999)-, el valor reversativo suele vincularse con casos de derivación verbal -o aplicación de un prefijo a una base verbal para formar un nuevo verbo (Varela y Martín García 1999; Martín García 2007)-. No obstante, existen casos que permiten una doble posibilidad interpretativa, tanto morfológica como semántica (Serrano Dolader 1999, 2011; Martín García 2007; RAE 2009). Así, desalar puede interpretarse como parasintético con valor privativo en desalar el agua del mar, o como derivado con valor reversativo en desalar el agua de las olivas; igualmente, desenterrar puede considerarse un parasintético con valor ablativo en desenterrar una planta, o un derivado con valor reversativo en desenterrar un tesoro (Grossmann 1994).

Esta caracterización de los reversativos plantea un inconveniente, pues no todos pueden vincularse con una acción previa, por lo que algunos autores se han visto en la necesidad de establecer grupos. Por ejemplo, Varela y Martín García (1999) diferencian entre verbos reversativos complejos, que entrañan la acción que denota la base (deshacer); y verbos reversativos no complejos, que no aluden a una acción previa (descrecer). Por su parte, Costa (2008) diferencia entre verbos reversivos, que presuponen no solo un estado sino una acción anterior (desabrochar); y verbos inversivos, que no presuponen una acción anterior (desarreglar).

Sin embargo, ni siquiera en los ejemplos más prototípicos concebimos necesariamente los reversativos como la inversión de la acción previa. Y, en todo caso, cabe preguntarse en qué consiste invertir la acción previa. En concreto, cuando pensamos en salir de un edificio no necesariamente imaginamos la acción de llevar a cabo el trayecto contrario al que recorrimos cuando entramos; se puede salir por una puerta distinta, por ejemplo. De la misma manera, cuando pensamos en descoser no imaginamos la acción de llevar a cabo los movimientos contrarios a aquellos que 
hacemos cuando cosemos; un vestido se puede descoser con unas tijeras, por un desgarrón, etc. (Cruse 1979; 1986).

Debido a los problemas de caracterización que puede generar la alusión a una acción previa, algunos autores prefieren vincular los reversativos con un estado anterior, rescatando así la idea inicial de Marchand (Cruse 1979; Horn 1988, 2002). Esto lleva a la necesidad de asumir que el valor reversativo surge cuando el prefijo, en este caso des, se une a bases télicas orientadas hacia la meta. Así, se diferencia del valor negativo, que surge cuando el prefijo se une a bases atélicas, que no desembocan en un estado resultante, como sucede en cuidar / descuidar -nótese que, en este caso, el verbo derivado se define como 'No cuidar de alguien o de algo, o no atenderlo con la diligencia debida' (DRAE 2001)-. Igualmente, el valor reversativo se diferencia del valor intensivo, que surge cuando el prefijo se une a bases télicas orientadas hacia el origen, como se observa en la pareja gastar / desgastar, donde el derivado no invierte el significado de la base, sino que lo intensifica (Vañó-Cerdá 1990; Battaner 1994; Varela y Martín García 1999; Martín García 2007; Serrano Dolader 2011).

Los verbos reversativos favorecen así un tipo de polaridad natural, o entropía, en el sentido de que el estado resultante al que da lugar el verbo derivado es más natural que el estado resultante al que dan lugar sus bases (Cruse 1979, 1986; Horn 1988, 2002). Es decir, el verbo base suele denotar una acción consciente, que impone un orden a algo, mientras que el verbo prefijado devuelve el tema -u objeto afectado- a su estado natural $^{3}$. Este carácter entrópico puede reflejarse en una serie de rasgos. Por ejemplo, base y derivado permiten la combinación con otra vez, sin que necesariamente se tenga que asumir que ni la acción que indica la base ni la acción que indica el derivado se hayan llevado a cabo más de una vez, como se observa en (1):

\footnotetext{
${ }^{3}$ En este sentido, el valor reversativo comparte con otros valores a los que pueden dar lugar los prefijos negativos el carácter [-loc], en la medida en que expresa también que el tema u objeto afectado abandona un punto, que puede ser físico -un lugar- o abstracto - un estado- (Lieber 2004). En el caso concreto del prefijo des-, su significado general ha sido definido como ‘valor egresivo’ (Battaner 1994; Costa 2008). Estas propuestas, en cierto modo, entroncan con la hipótesis localista de Lyons (1977).
} 
(1) Colgamos el cuadro en la pared para ver cómo quedaba y lo descolgamos otra vez porque no iba bien con el estilo del salón.

Asimismo, en muchos casos, solo el verbo derivado puede aparecer en la construcción incoativa con se-como demuestra el perfecto funcionamiento de (2), frente a la anomalía de (3)-, lo que explicita que, mientras que el estado resultante de la base exige algún tipo de energía para poder llevarse a cabo, el estado resultante del derivado se ve favorecido por la inercia, o la tendencia general del universo:

(2) El cuadro se descolgó.

(3) \# El cuadro se colgó.

La caracterización de los reversativos en función de su adhesión a bases télicas puede llevarnos a pensar, por simetría, que el derivado es también télico. No obstante, la telicidad tampoco es un concepto exento de controversia. Por ejemplo, se suele plantear si el carácter télico de un predicado es un rasgo convencional o un efecto composicional. En este sentido, Horn $(1988,2002)$ señala un dato interesante con respecto a los reversativos en inglés: muchos de los verbos prefijados con un- parecen oponerse a verbos con partículas, más que a las bases simples; así, unbutton ['desabotonar'] parece contraer una relación contrastiva con button up ['abotonar completamente'], más que con button ['abotonar']. El autor utiliza el paralelismo como evidencia para destacar el carácter entrópico de los verbos reversativos, pues la partícula up expresa ‘compresión’, mientras que un- transmitiría ‘dispersión’ o ‘expansión’. Sin embargo, este hecho puede servir también de pista para desvelar el funcionamiento de la telicidad en algunos verbos reversativos.

Como se mostrará en los siguientes apartados, la pragmática contribuye a perfilar el tipo de oposición entre los estados resultantes de base y derivado, y esto se refleja en la imagen aspectual del predicado derivado. 


\section{Escalaridad y aspecto: visiones y revisiones}

El aspecto nos permite describir la estructura temporal interna de un evento; es decir, convoca la imagen mental que tenemos de cómo se lleva a cabo, cómo está dispuesta, o cómo concebimos una situación. Desde Vendler (1957), se han establecido cuatro clases aspectuales, o cuatro esquemas que deberían servir como focos para clasificar los distintos tipos de eventos: estados, actividades, realizaciones y logros. Estas clases aspectuales se diferencian en función de tres criterios: duración, cambio y delimitación o telicidad (Dowty 1979; De Miguel 1999; Kearns 2000). Los estados son eventos durativos, pues se extienden en el tiempo; no reflejan ningún tipo de cambio, por lo que se consideran homogéneos; y no están delimitados, en el sentido de que no están orientados hacia un punto final. Un ejemplo de estado sería saber polaco. Las actividades son eventos durativos, que también se extienden en el tiempo; implican cambio, por lo que son eventos heterogéneos; y no están orientadas hacia un punto final: una porción del evento cuenta como el evento mismo. Un ejemplo de actividad sería caminar. En este sentido, si alguien está caminando y el evento se interrumpe, puede decirse que ha caminado. Las realizaciones son eventos durativos, porque se extienden en el tiempo; al igual que las actividades, también implican cambio; pero, a diferencia de estas, las realizaciones son delimitadas, en el sentido de que están orientadas hacia un punto final. Un ejemplo de realización sería leer un libro: en este caso una porción del evento no cuenta como el evento mismo; o, dicho de otra manera, si alguien está leyendo un libro y el evento se interrumpe, no puede decirse que ha leído un libro. Finalmente, los logros son eventos que carecen de duración, pues no se extienden en el tiempo; al contrario, se trata de eventos puntuales; e implican cambio, pues su razón de ser se basa precisamente en expresar un cambio de estado. Un ejemplo de logro sería alcanzar la cima.

Tras la clasificación aspectual de Vendler, dos son las preguntas en torno a las cuales gira buena parte del debate lingüístico sobre el aspecto (cf. Rappaport-Hovav 2008; Rothstein 2012). En primer lugar, cabe preguntarse a qué se pueden aplicar estas clases aspectuales. Parece ya demostrado que no solo los verbos influyen en la determinación de la clase aspectual: diversos componentes del predicado pueden 
contribuir a dibujar la imagen del evento. Así, si bien leer un libro se entiende como una realización, leer contaría como una actividad. Igualmente, mientras que caminar se considera una actividad, caminar hasta la esquina se asume como una realización. Efectivamente, la existencia de constituyentes que delimiten el evento, como un complemento directo delimitado, o un sintagma preposicional que acote la cantidad de movimiento expresada en el verbo, pueden bastar para convertir una actividad en una realización. Pero no solo los componentes del predicado pueden influir en la clase aspectual. Si alcanzar la cima se considera un evento puntual en Juan alcanzó la cima a las seis, este mismo verbo puede transmitir una imagen distinta con un sujeto diferente, como sucede en Miles de turistas alcanzaron la cima durante el verano. En efecto, no solo los componentes del predicado, sino de la oración en general, contribuyen a perfilar la imagen aspectual del evento. Es lo que define la naturaleza composicional del Aktionsart, o aspecto léxico. No obstante, lo que clasificamos aspectualmente tampoco son las oraciones, pues estas no son más que el reflejo de la manera en que concebimos las situaciones (Declerck 1979).

La segunda pregunta tiene que ver con el número de clases que establece Vendler: ¿Son efectivamente cuatro? ¿Son más? ¿Son menos? Más allá de las modificaciones en la definición y comportamiento de los estados, y de la pertinencia o no de considerar los semelfactivos ${ }^{4}$ como un tipo aspectual diferente, la posible desestabilización de la clasificación aspectual vendleriana que nos interesa para los propósitos de este artículo tiene que ver con las interacciones entre la tríada de los predicados dinámicos actividades - realizaciones - logros.

En efecto, existe cierta controversia sobre si hay una distinción clara entre las realizaciones y los logros; en concreto, se ha puesto en tela de juicio el estatuto de estos últimos como categoría, ya que se asume que, si denotan cambio, no pueden ser puntuales (pues exigen, al menos, dos momentos), por lo que en ocasiones se los ha

\footnotetext{
${ }^{4}$ Los semelfactivos serían predicados puntuales que no suponen un cambio de estado, como rap the table ['dar un golpe en la mesa'] (Kearns 2000) o dar un salto (De Miguel 1999). Para algunos autores, constituirían un ejemplo de aspectualidad cuantitativa, en la medida en que el evento se da de forma única (De Miguel 1999).
} 
considerado realizaciones muy breves. Por ejemplo, para Pustejovsky ([1991]2005), no es necesario considerar las realizaciones y los logros como dos clases aspectuales: ambos serían transiciones, y la única diferencia sería que las primeras reflejarían actividad y cambio, mientras que los segundos no harían referencia explícita a la actividad. Por el contrario, para otros autores, como Piñón (1997), los logros constituyen un tipo de evento diferente, caracterizado por su naturaleza instantánea: reflejan justo la frontera del cambio de estado, y se insertan como principios o finales de eventos más amplios.

Si existe tensión en la frontera entre realizaciones y logros, el territorio que media entre las actividades y las realizaciones no es menos polémico. En este sentido, se ha planteado si las realizaciones son, en realidad, un tipo ontológico de evento o si son el resultado composicional de diversos factores. Así, para Rothstein (2012), las actividades se caracterizan léxicamente por su homogeneidad incremental: implican cambio, pero se trata de un cambio homogéneo ${ }^{5}$; se establecen como evento en cuanto son prominentes y se mantienen como tales a lo largo de períodos de tiempo incremental. En cuanto a las realizaciones, están constituidas por un componente actividad que, a su vez, está estructurado por un componente de cambio de estado (que orienta la actividad hacia un punto final). Desde esta perspectiva, la autora establece tres tipos de realizaciones, en función del componente actividad que intervenga: a) realizaciones en las que el componente actividad está lexicalizado, que son las que pasan fácilmente a actividades cuando no aparece el complemento directo (leer un libro); b) realizaciones en las que el componente actividad no está lexicalizado, sino que es un predicado general que identificamos de manera prototípica (construir una casa); c) realizaciones en las que el componente actividad tampoco está lexicalizado y que, además, se caracterizan por un cambio repentino de estado (abrir la puerta).

\footnotetext{
${ }^{5}$ En este sentido, las actividades son heterogéneas frente a los estados (que son homogéneos), pues las primeras implican cambio y los segundos no. Igualmente, las actividades son homogéneas frente a las realizaciones (que son heterogéneas), pues las primeras implican un cambio idéntico en todas sus fases, mientras que las segundas indican un cambio orientado hacia un punto final. En este sentido, la homogeneidad o heterogeneidad sería una cuestión de perspectiva -amplia o restringida- (Kearns 2000).
} 
Para otros autores, las realizaciones son el resultado de un efecto composicional. Esta propuesta se ha defendido, en buena medida, desde la perspectiva escalar. En efecto, en las últimas décadas (cf. Demonte y McNally 2012), una parte importante de los estudios sobre el aspecto se ha relacionado con la escalaridad, en parte vinculada al concepto de incrementalidad (Dowty 1991; Tenny 1995; Krifka 1998).

La incrementalidad se basa en el homomorfismo entre la estructura del argumento tema -u objeto afectado- y la estructura del evento (Dowty 1991). Así, adaptando el ejemplo de Dowty, si alguien le encarga al jardinero que corte el césped ['mow the lawn'], y al cabo de un rato vuelve, puede observar cómo ha progresado el evento a partir del estado del césped. Para que se lleve a cabo esta relación mereológica, es necesario que uno de los argumentos del predicado esté estructurado en partes (Krifka 1998). Esto es lo que permite que el evento progrese a la vez que el argumento se ve afectado, consumido, construido o destruido. Así, en ejemplos como comer(se) una manzana o dibujar un círculo, cada parte del evento se corresponde con una parte de la manzana o del círculo, respectivamente; frente a lo que ocurre en casos como hacer un punto, que no permiten la relación mereológica, pues el objeto no se puede estructurar en partes.

La incrementalidad constituye, por tanto, un caso concreto de organización escalar del evento. La habilidad para entender y usar escalas es una parte fundamental de nuestra capacidad cognitiva (Tenny 1995), por lo que no es de extrañar que esté presente en el significado de los verbos. Para Tenny, existen tres tipos de verbos en función de cómo la noción de medida interactúa con tres tipos de escalas:

a) Predicados de tema incremental. En ellos la escala se proyecta sobre la extensión o el volumen del tema u objeto afectado, que es graduable. La implicación del objeto en el evento puede ser un tipo de creación, consumo, afección, etc., como en escribir una carta, beber una cerveza, pintar una pared.

b) Predicados de cambio de estado. Aquí podrían incluirse diversos tipos de verbos, como los verbos de cambio de estado deadjetivales (secar, suavizar) o los verbos de 
cambio de estado concretados en participios (hervir, arreglar) ${ }^{6}$. En estos casos, la escala se proyecta sobre la propiedad concreta en la que cambia el tema u objeto afectado; por ejemplo, el grado de sequedad, suavidad, etc. Para Tenny, los predicados de tema incremental y los predicados de cambio de estado son muy semejantes, en la medida en que ambos implican un cambio de estado en el objeto afectado ${ }^{7}$.

c) Predicados de movimiento. En este caso, la escala se proyecta sobre la distancia. Aquí podrían incluirse tanto verbos de movimiento dirigido (ascender), como verbos de manera de movimiento más un sintagma que acote la cantidad de desplazamiento que se ha llevado a cabo (caminar hasta la esquina).

En realidad, todos los verbos de cambio pueden estar asociados a una escala, aunque esa escala no necesariamente ha de venir codificada en el verbo, sino que puede ser aportada por algún otro constituyente (Beavers 2008; Rappaport-Hovav 2008). Por ejemplo, en los verbos de movimiento dirigido y en los verbos de cambio de estado deadjetivales, la escala la aporta el propio el verbo. Piénsese, por ejemplo, en estos últimos, donde el verbo tiene un comportamiento paralelo al tipo de escala que constituye el adjetivo (Kennedy y McNally 2005) ${ }^{8}$ : seco $>$ secar; largo $>$ alargar. Por el contrario, en otros casos, la escala es convocada por algún otro elemento. Así, para que los verbos de manera de movimiento constituyan un evento escalar necesitan un

\footnotetext{
${ }^{6}$ Ambos son clasificados como verbos de cambio de estado por Demonte (1994). En este sentido, Koontz-Garboden y Levin (2005) establecen diferencias entre dos tipos de predicados de cambio de estado, en función de dos tipos de estado: aquellos que derivan de propiedades -donde el estado da lugar al predicado- (que serían morfológicamente deadjetivales), y los que derivan de estados resultantes -donde el predicado da lugar al estado- (y dicho estado se concretaría en el participio).

${ }^{7}$ En realidad, Tenny incluye en este apartado predicados con construcciones resultativas en inglés, como Wash the floor clean, donde la escala la aportaría la propia estructura resultativa, a partir de la propiedad de la limpieza. Este tipo de estructuras no pueden darse en español, al menos en una misma predicación, por lo que solo se admitirían paráfrasis como Limpió el suelo hasta que quedó limpio.

${ }^{8}$ El tipo de escala que convoca el adjetivo puede influir también en el funcionamiento de otros tipos de predicados: por ejemplo, puede explicar el comportamiento aspectual de verbos semicopulativos como volver (Batiukova, en prensa).
} 
sintagma que refleje una trayectoria delimitada y que, al mismo tiempo, pueda desplegar una escala en función del acercamiento progresivo a la meta. Igualmente, en los predicados de tema incremental, es el tema u objeto afectado el que convoca la escala (Kennedy 2012) ${ }^{9}$.

Igualmente, las escalas pueden ser de dos tipos (Beavers 2008; RappaportHovav 2008): de dos puntos y de varios puntos. Las escalas de dos puntos denotan un cambio entre dos estados complementarios. Un ejemplo en el ámbito del movimiento sería el del verbo salir; en el plano más abstracto del cambio de estado, un ejemplo sería morir. Las escalas de varios puntos reflejan un cambio de estado que permite puntos intermedios, a lo largo de los cuales el tema avanza gradual o progresivamente, como sucede en ascender (movimiento), adelgazar (cambio de estado), leer un libro (tema incremental).

Los dos tipos de escalas se comportan de manera diferente desde el punto de vista aspectual (Rappaport-Hovav 2008). Cuando el verbo está asociado a una escala de dos puntos, se produce una transición completa de un estado a otro, de ahí la anomalía de (4):

(4) \# Juan salió, pero no del todo / pero no completamente.

Cuando el verbo está asociado a una escala de varios puntos, se entraña algún tipo de cambio a lo largo de la escala, pero el cambio completo no es más que una inferencia pragmática, por lo que puede ser cancelada. Así sucede con los predicados de tema incremental, como en (5); con los verbos de cambio de estado deadjetivales que constituyen logros de grado o predicados de compleción gradual, como en (6); y con los verbos de cambio de localización con una escala de trayectoria, como en (7):

(5) I mowed the lawn, but not all of it.

['Corté el césped, pero no del todo / no completamente / no todo’]

\footnotetext{
${ }^{9}$ En el modelo de Rappaport-Hovav y Levin, los verbos de manera son no escalares, mientras que los verbos de resultado son escalares. Eso no quiere decir que los primeros no puedan convertirse en predicados escalares composicionalmente (Rappaport-Hovav y Levin 2010).
} 
(6) If you put the tomatoes out on the porch, the sun will ripen them a bit.

['Si pones los tomates en el porche, el sol los madurará un poco’]

(7) I threw the ball to Mary, but it didn’t get there.

['Le lancé la pelota a María, pero no llegó allí’]

(Rappaport-Hovav 2008).

En definitiva, la lectura télica, o "lectura completamente", es fruto de una implicatura conversacional generalizada, debida a un principio conversacional de refuerzo -el Principio de Informatividad (Levinson 2000) o el Principio de Relación (Horn [1984] 1998) ${ }^{10}$-, que nos insta a buscar una interpretación más informativa para algunas formas débiles (Atlas y Levinson 1981; Hay, Kennedy y Levin 1999; Jeschull 2007) ${ }^{11}$.

Como se demostrará en el siguiente apartado, en muchas ocasiones la interpretación télica de los verbos reversativos será el resultado de una implicatura conversacional generalizada, basada en el refuerzo pragmático de formas débiles. Las inferencias sistemáticas contribuyen también, pues, a perfilar la imagen aspectual de algunos verbos.

\footnotetext{
${ }^{10}$ El Principio de Informatividad, en el modelo de Levinson, interactúa con el Principio de Manera y con el Principio de Cantidad. El Principio de Relación, en el modelo de Horn, mantiene una tensión dialéctica o antinómica exclusivamente con el Principio de Cantidad. En ambos modelos, se trata de un principio de refuerzo; la distinción entre ambas propuestas reside en si se considera que la marcación formal, o la Manera, puede constituir un principio conversacional con fuerza de heurística (Levinson), o si queda escindida entre el Principio de Cantidad y el Principio de Relación (Horn). Para una comparación entre ambos modelos, puede verse Rodríguez Rosique (2008).

${ }^{11}$ En este sentido, algunos autores (Declerck 1979) consideran que la (de)limitación o (a)telicidad no es una oposición bipolar, sino tripartita, de forma que clasifican los eventos como +delimitados, delimitados, $\varnothing$ delimitados. Este último grupo sería neutral con respecto a la (de)limitación, y vendría a explicitar la existencia de formas débiles. Asimismo, Olsen (1994) considera que los predicados -télicos, frente a los +télicos (más estables), suelen poder interpretarse contextualmente como +télicos. En este sentido, los primeros serían débiles con respecto a los segundos, y susceptibles, por ello, de sufrir un refuerzo pragmático.
} 


\section{Un tipo de oposición "muy verbal”}

Como se ha comentado, los verbos reversativos son formas marcadas, pues el estado resultante del predicado derivado remite al estado resultante del predicado base. Asimismo, el tipo de oposición que se establece entre los estados resultantes de base y derivado se refleja en la imagen aspectual del verbo reversativo ${ }^{12}$. En este sentido, se pueden establecer tres tipos de reversativos.

4.1. Reversativos que reflejan una relación de oposición complementaria entre el estado resultante de la base y el estado resultante del derivado.

(8) La Policía colombiana desactivó ayer un cinturón-bomba colocado a la madre de la gerente de una cadena de supermercados en la ciudad de Manizales.

(RAE, CREA, La voz de Galicia, 18/12/2000).

En este caso, el reversativo constituye un predicado télico, como demuestra su comportamiento ante las distintas pruebas aspectuales (Dowty 1979; De Miguel 1999; Kearns 2000). En concreto, es anómalo al combinarse con adverbios durativos, como durante (9); funciona perfectamente con sintagmas y estructuras que indican lapso temporal $(10,11)$; y, como predice la paradoja imperfectiva, la forma en progresivo no entraña la forma en perfecto (12):

(9) \# La policía colombiana desactivó un cinturón-bomba durante diez minutos.

(10) La policía colombiana desactivó un cinturón-bomba en diez minutos.

(11) ¿Cuánto tardó la policía en desactivar un cinturón-bomba?

\footnotetext{
${ }^{12}$ Cruse (1986) y Grossmann (1994) ya hablan de dos tipos de reversativos, en función de que denoten un cambio absoluto o un cambio relativo.
} 
(12) La policía está desactivando un cinturón bomba \# La policía ha desactivado un cinturón-bomba.

Este tipo de predicados es también compatible con complementos temporales que indican el instante concreto en el que se lleva a cabo el evento, por lo que tienen un comportamiento muy similar a los logros (13):

(13) Novales introdujo su tarjeta en el escaño a las 9.28 horas, dos minutos antes de que comenzara el pleno, pero la desactivó a las 10.24.

(RAE, CREA, La voz de Galicia, 29/12/2004)

En este sentido, se pueden observar diferencias entre (8) e (13) con respecto a algunas pruebas (Piñón 1997; De Miguel 1999). En (8), la combinación con casi puede admitir una doble interpretación, como se observa en (14): a) el evento comenzó, pero no acabó; b) el evento no comenzó ${ }^{13}$. En (13), sin embargo, parece que solo la segunda interpretación es posible, como refleja (15):

(14) La policía colombiana casi desactivó un cinturón-bomba.

(15) Novales casi desactivó su tarjeta a las 10.24.

Igualmente, parece haber diferencias en el grado de aceptabilidad de (8) y (13) al combinarse con terminar de:

(16) La policía colombiana terminó de desactivar un cinturón-bomba.

(17) \# Novales terminó de desactivar su tarjeta a las 10.24.

No obstante, nótese que los logros pueden constituir principios o finales de eventos mayores y, quizás, lo que se termina en (16), o lo que empieza pero no se termina en (14), no es el cambio mismo de un estado (activado) a otro (desactivado), sino las operaciones que desembocan en este cambio ${ }^{14}$.

\footnotetext{
${ }^{13}$ La doble interpretación de casi en las realizaciones demostraría que los eventos están compuestos de fases, por lo que el adverbio podría afectar a la actividad o al estado resultante (Pustejovsky [1991] 2005).

14 Nótese que desactivar podría combinarse igualmente con adverbios de intención y manera (cuidadosamente, intencionadamente, etc.), algo que no suelen admitir los logros (Piñón 1997).
} 
En este caso, los estados resultantes a los que dan lugar la base y el derivado mantienen una relación de oposición complementaria (Lyons 1977; Cruse 1986; Grossmann 1994; Paradis 2001; Kennedy y McNally 2005). En concreto, ambos estados dividen de manera exhaustiva un parámetro semántico, por lo que (18) es una contradicción, y (19) es una tautología. Igualmente, este parámetro no es graduable (20). Únicamente permite adverbios que indican el carácter cerrado de la escala que convocan, como demuestra la combinación con completamente en (21):

(18) \# El cinturón-bomba no está ni activado ni desactivado.

(19) \# El cinturón-bomba está activado o desactivado.

(20) \# El cinturón-bomba está \{muy / poco / un poco / bastante\} desactivado.

(21) El cinturón-bomba está completamente desactivado.

Desactivar es un verbo reversativo formado por derivación; en concreto, se crea mediante la adhesión del prefijo des- a la base verbal activar. A su vez, activar es un verbo deadjetival, formado por la adhesión del sufijo -ar a la base adjetival activo ${ }^{15}$. Como se comentó, los verbos deadjetivales reflejan claramente el cambio escalar a partir de la escala que convoca el adjetivo. En este sentido, tal como se ha mostrado, activo / no activo convoca una escala de dos puntos.

No obstante, cabe preguntarse si este análisis es extensible a otros verbos reversativos cuyas bases surgen mediante otros procedimientos, pero que exhiben el mismo tipo de oposición entre los estados resultantes. Piénsese, por ejemplo, en descolgar:

\footnotetext{
${ }^{15}$ El sufijo -ar es uno de los sufijos verbalizadores más neutros en cuanto a su significado se refiere, y puede convocar una gran variedad de valores (Rifón 1997). Nótese que la verbalización deadjetival se ha entendido como un mecanismo que permite fusionar una relación de atribución, en el sentido de orientar una propiedad a una base; a partir de ese significado general, se podrían establecer diversos subgrupos de verbos deadjetivales, en función de las peculiaridades de esa relación de atribución (Cifuentes Honrubia 2011).
} 
(22) El ladrón descolgó el cuadro de la pared siguiendo la sugerencia de una asistente de la galería de arte.

(RAE, CREA, Clarin, 07/03/1997)

El DRAE (2001) ofrece como primera definición de este verbo 'Bajar lo que está colgado’. En concreto, descolgar es un verbo reversativo que surge por la adhesión del prefijo des- a la base colgar, que, a su vez, deriva del latín collocāre ${ }^{16}$. Asimismo, la base de datos ADESSE lo califica como "verbo de localización”, formando paradigma con quitar y poner. Se puede entender, por tanto, como un verbo que indica cambio de posición ${ }^{17}$ y que, en términos de oposición, refleja el paso de “estar en X” a "no estar en X”. Los estados resultantes son, por tanto, complementarios, como demuestra la contradicción de (23), la tautología de (24), y la anomalía de (25):

(23) \# El cuadro no está ni colgado ni descolgado.

(24) \# El cuadro está colgado o descolgado.

(25) \# El cuadro está \{muy / poco / un poco / bastante $\}$ descolgado ${ }^{18}$.

Otro caso que refleja el mismo tipo de oposición entre los estados resultantes de base y derivado es el de verbos como desenchufar, aunque la explicación puede ser menos transparente, por la propia naturaleza del verbo.

\footnotetext{
${ }^{16}$ A su vez, algunos autores señalan que el verbo collocāre permitiría una amalgama latina de base, del tipo ‘poner en un lugar' (Cifuentes Honrubia 1999).

${ }^{17}$ No obstante, el verbo no tiene por qué denotar únicamente cambio de posición. Y la orientación vertical (Cifuentes Honrubia 1999) de descolgar puede traducirse en otros valores, no necesariamente puntuales, sino durativos, como se observa en los siguientes ejemplos: “[...] lo que le permitió ver cómo se descolgaba una inmensa pancarta con la leyenda 'Felicidades, presidente'” (RAE, CREA, La Vanguardia, 03/04/1995); "El detenido se había evadido el 20 de octubre por el clásico método de descolgarse por unas sábanas desde lo alto de una tapia para alcanzar la calle” (RAE, CREA, El Diario Vasco, 31/01/2011).

${ }^{18}$ Una estructura del tipo El cuadro está un poco descolgado podría interpretarse como que está "un poco ladeado”; es decir, en este caso, el cuantificador más bien funcionaría como atenuativo (De Miguel 1999).
} 
(26) En caso de tormenta, desconectar la antena y desenchufar el aparato de la red.

(RAE, CREA, Instrucciones impresas 1998)

El DRAE (2001) define desenchufar como 'Separar o desacoplar lo que está enchufado'. Se trata de un verbo reversativo formado por la adhesión del prefijo des- a la base verbal enchufar. A su vez, la base constituye un verbo denominal, formado por la unión del sufijo verbalizador -ar a la base sustantiva enchufe. ADESSE lo califica como un verbo de unión, que conforma paradigma con separar. Más allá de la incorporación o fusión del sustantivo enchufe, y sin entrar en los procesos semánticos que ello supone, el verbo denominal desencadena un cambio de estado en el nuevo tema u objeto afectado (en el ejemplo anterior, el aparato), que pasa del estado "enchufado" al estado "desenchufado" ${ }^{19}$. De nuevo, esta transición refleja un cambio completo, pues los estados resultantes mantienen una relación de oposición complementaria; así, (27) es una contradicción, (28) es una tautología, y (29) refleja la imposibilidad de graduar la transición de un estado a otro:

(27) \# El aparato no está ni enchufado ni desenchufado.

(28) \# El aparato está enchufado o desenchufado.

(29) \# El aparato está \{muy / poco / un poco\} desenchufado.

En definitiva, en estos casos el objeto afectado pasa del estado al que ha dado lugar la base a un estado anterior, originario, o más natural. La relación entre estos estados resultantes es de oposición complementaria. Los casos más claros son aquellos en los que la base inicial es un adjetivo, pues la estructura escalar es más nítida; sin embargo, aquellos vinculados de alguna manera con el cambio de posición pueden ofrecer un comportamiento similar.

\footnotetext{
${ }^{19}$ Para el comportamiento, constitución y valores semánticos de los verbos causativos denominales puede verse Lavale Ortiz (2007).
} 
4.2. Reversativos que reflejan una relación de oposición antonímica ${ }_{1}$ entre el estado resultante de la base y el estado resultante del derivado

(30) Esa noche deshinchamos las ruedas de los jeeps como represalia.

(RAE, CREA, M. Leguineche, El camino más corto, 1995)

Predicados como el que aparece en (30) admiten la combinación con sintagmas y estructuras que denotan lapso temporal, como se observa en (31) y (32), con la interpretación télica de "transición completa”:

(31) Deshinchamos las ruedas en diez minutos.

(32) ¿Cuánto tiempo tardamos en deshinchar las ruedas?

No obstante, este valor télico, o transición completa, está desencadenado pragmáticamente, como demuestra el hecho de que pueda cancelarse o bloquearse contextualmente:

(33) Esa noche deshinchamos las ruedas, pero no del todo.

(34) Esa noche deshinchamos un poco las ruedas de los jeeps.

Como planteaba Tenny (1995), los predicados de tema incremental y los predicados de cambio de estado se asemejan bastante, pues ambos reflejan un cambio de estado en el objeto afectado o tema. Para algunos autores, la diferencia estriba en qué elemento convoca la escala: el propio verbo o el objeto afectado (Hay, Kennedy y Levin 1999; Rappaport-Hovav 2008; Kennedy 2012). Ejemplos como el de (30) pueden resultar ambiguos, pues, por un lado, permiten una lectura mereológica, en el sentido de que el evento de deshinchar progresa según se van viendo afectadas las ruedas. No obstante, también resulta revelador el comportamiento de este predicado con casi, pues no parece admitir una lectura en la que el evento se inicia pero no se completa, sino que más bien parece apuntar a que el inicio no llegó a comenzar:

(35) Esa noche casi deshinchamos las ruedas de los jeeps.

Y, en este sentido, si estamos deshinchando las ruedas, ya las hemos deshinchado (un poco). 
Los estados resultantes a los que dan lugar tanto el predicado base (hinchar las ruedas) como el derivado (deshinchar las ruedas) representan en este caso una relación de oposición antonímica (Lyons 1977; Cruse 1986), pues hinchadas / deshinchadas no agotan el parámetro semántico que denotan; más bien, constituyen los extremos de una escala, que es perfectamente graduable:

(36) Las ruedas estaban \{un poco / muy / bastante / demasiado\} deshinchadas. No obstante, se trata de una escala cerrada (Paradis 2001, Kennedy y McNally 2005), por eso se puede activar la implicatura conversacional de refuerzo, y por eso se puede explicitar, pues ambos extremos pueden quedar delimitados mediante completamente:

(37) Las ruedas estaban completamente deshinchadas.

Un caso semejante sería el de descorrer, que tendemos a interpretar télicamente, en el sentido de transición completa:

(38) Al descorrer la tela aplaudíamos y le dábamos al monigote un sentido diferente al de su historia real.

(RAE, CREA, A. Jodorowsky, La danza de la realidad, 2001)

Sin embargo, este valor no es más que una implicatura conversacional que, como tal, puede ser explicitada (39) o cancelada (40):

(39) Todas las mañanas, a las once, gracias a su llave maestra, entraba en su habitación, “inocentemente”, se dirigía a la ventana, descorría las cortinas de par en par.

(RAE, CREA, F. Arrabal, La torre herida por el rayo, 1983)

(40) La señora recogió los platos, pasaron a la sala y Heberto descorrió un poco la cortina para mirar a la calle.

(RAE, CREA, S. Gamboa, Páginas de vuelta, 1998)

Lo importante de este grupo de reversativos es que la interpretación télica, o la lectura de transición completa, no es más que una implicatura conversacional generalizada y que, por ello, puede ser cancelada o bloqueada en el contexto. Este tipo de significado es 
tan sistemático que puede llegar a confundirse con el significado convencional. Sin embargo, forma parte de la pragmática; en concreto, está vinculado con la tendencia general de reforzar enunciados débiles, hasta llegar a una interpretación más fuerte o específica, y, en el caso concreto de los enunciados contrastivos, puede reforzarse hasta conseguir una interpretación contraria. Aunque todavía lo barajamos como hipótesis, probablemente el tipo más común de reversativos se incluya dentro de este grupo.

4.3. Reversativos que reflejan una relación de oposición antonímica ${ }_{2}$ entre el estado inicial y el estado resultante del derivado

(41) Parece que ha decidido desacelerar su carrera cinematográfica...

(RAE, CREA, La voz de Galicia, 15/01/2004)

(42) La planificación y las diversas zonas permiten contar con productos frescos y no tener stock, que desmejora la calidad del producto.

(RAE, CREA, Revista Supercampo. 2000)

Estos predicados son compatibles tanto con adverbios durativos (43), como con sintagmas o estructuras que indican lapso temporal $(44,45)$, aunque con distinta interpretación; en el primer caso se consideran predicados atélicos, y en el segundo indican una cantidad de cambio delimitada:

(43) Desaceleró su carrera cinematográfica durante dos años

(44) ¿Cuánto tiempo tardó en desacelerar su carrera cinematográfica?

(45) Desaceleró su carrera cinematográfica en dos años

Casos como desacelerar o desmejorar constituyen verbos de cambio de estado; en concreto, forman parte de los denominados logros de grado (Hay, Kennedy y Levin 1999) o verbos de compleción gradual (Bertinetto y Squartini 1995). Este tipo de verbos pueden ser morfológicamente deadjetivales (desmejorar), o estar vinculados en algún sentido con un adjetivo (desacelerar) (Bertinetto y Squartini 1995). Se caracterizan por 
tener un tipo de "telicidad variable”: pueden ser logros (si denotan un cambio mínimo), actividades (si denotan un cambio no delimitado) o realizaciones (si denotan un cambio delimitado $)^{20}$. En estos casos, la imagen escalar del verbo está vinculada con la escala del adjetivo que toman como base.

La oposición entre los estados inicial y final del tema es de nuevo de carácter antonímico, por lo que el estado al que da lugar el reversativo es perfectamente graduable:

(46) Su carrera está \{poco / un poco / muy / bastante\} desacelerada.

La relación escalar en estos casos es más compleja que en los comentados anteriormente. Quizás se complica más porque este tipo de verbos deadjetivales ya denotan “funciones de medida de cambio” (Hay, Kennedy y Levin 1999), que, además, establecen vinculaciones semánticas con pares opuestos.

Los reversativos de este grupo también difieren de los del grupo anterior en que los estados resultantes se sitúan en escalas abiertas, que no tienen un límite (Paradis 2001; Kennedy y McNally 2005). No obstante, ese límite puede venir dado composicionalmente, por elementos que cuantifican la cantidad de cambio implicada en el evento:

(47) En los años treinta la ciudad desaceleró su crecimiento demográfico al 3,4\% anual.

(RAE, CREA, F. Puyo, Bogotá, 1992)

Nótese que no hay un grado de “completamente desacelerado” que sea igual al estatismo; de hecho, existen unidades léxicamente más informativas que suelen emplearse cuando queremos denotar ese grado cero:

(48) [Las tasas de interés muy elevadas] tienden a desacelerar e incluso paralizar múltiples actividades económicas.

\footnotetext{
${ }^{20}$ Para algunos autores, la lectura de cambio mínimo de estos eventos -es decir, su interpretación como logros- sería un tipo de telicidad (Bertinetto y Squartini 1995; Kearns 2007); para otros (Hay, Kennedy y Levin 1999), la lectura télica sería la lectura en la que el cambio se lleva a cabo “completamente”, bien porque se refuerza pragmáticamente como una implicatura, o porque el cambio está acotado composicionalmente mediante un sintagma.
} 
(RAE, CREA, El Universal, 21/04/1993).

Si bien es cierto que modificadores como totalmente o completamente pueden aparecer con estados situados en escalas abiertas, como desacelerado, en estos casos los adverbios se entenderían más como un procedimiento de intensificación que como un procedimiento de cuantificación o delimitación escalar (Kennedy y McNally 2005; Kearns 2007), como se observa en (49):

(49) Entonces con un ritmo totalmente desacelerado me desprendí de la ropa que llevaba encima, me guardé en la cama y apagué la luz ${ }^{21}$.

(50) Ciudades en donde la población crecía a un ritmo completamente desacelerado ${ }^{22}$.

\section{Conclusión}

En definitiva, los verbos reversativos morfológicos no constituyen una clase homogénea en términos aspectuales. Efectivamente, en todos ellos puede decirse que des- se adhiere a una base télica, o, al menos, que el prefijo impone un límite a la base que constituye el punto de partida para la oposición. No obstante, se diferencian en la imagen aspectual que convocan o en cómo adquieren esa telicidad. En concreto, provisionalmente se han establecido tres grupos de reversativos: reversativos télicos, vinculados con una escala de dos puntos, que expresan una transición entre estados resultantes complementarios; reversativos pragmáticamente télicos, vinculados con escalas de varios puntos, que expresan una transición entre estados resultantes antónimos: como convocan escalas cerradas, permiten que la lectura de transición completa se desencadene en forma de refuerzo pragmático o implicatura conversacional; y reversativos generalmente atélicos (en el sentido de transición completa), vinculados con escalas de varios puntos, abiertas,

\footnotetext{
${ }^{21}$ Ejemplo extraído de Google <soyunartista.blogspot.com>

${ }^{22}$ Ejemplo extraído de Youtube <www.youtube.com [El cohete rojo]>
} 
que no obstante pueden denotar una cantidad concreta de cambio de forma externa o composicional.

Aunque esta clasificación se presenta como hipótesis de trabajo, permite observar la intensa relación que esta clase de verbos establece entre oposición, escalaridad y aspecto.

En sentido general, los verbos reversativos, como especies negativas, constituyen formas marcadas, pues remiten a un estado (resultante) anterior. Precisamente la relación de oposición que se establece entre los estados inicial (generalmente desencadenado por la base) y final (desencadenado por el derivado) se refleja en la imagen aspectual del verbo reversativo. Asimismo, un grupo importante de estos verbos refuerza el tipo de oposición entre el estado resultante de la base y el estado resultante del derivado a través de una implicatura conversacional generalizada, lo que constituye un nuevo ejemplo del papel que desempeñan las inferencias pragmáticas en el funcionamiento de las categorías verbales, o, en sentido amplio, de las categorías gramaticales.

Bibliografía

ADESSE. Base de datos de Verbos, Alternancias de Diátesis y Esquemas SintácticoSemánticos del Español, Universidad de Vigo.

Atlas, J. y Levinson, S. 1981. It-clefts, informativeness, and logical form: Radical pragmatics. P. Cole (ed.), Radical Pragmatics, Nueva York: Academic Press, 1-61.

Batiukova, O. En prensa. “Sobre la escalaridad de los verbos de movimiento”. Actas del X Congreso de Lingüistica General, Zaragoza, 2012.

Battaner, M. P. 1996. Características léxico-semánticas de los verbos prefijados con des- en DRAE 1992. Boletín de la Real Academia Española, LXXVI-CCLXIX, 309-370.

Beavers, J. 2008. Scalar complexity and the structure of events. J. Dölling, T. HeydeZybatow y M. Schäfer (eds.), Event Structures in Linguistic Form and Interpretation, Berlin: Mouton de Gruyter, 245-265. 
Bertinetto, P. M. y Squartini, M. 1995. An attempt at defining the class of 'gradual completion verbs'. P. M. Bertinetto, V. Bianchi, J. Higginbotham y M. Squaritini (eds.), Temporal Reference, Aspect and Actionality Vol. I: Semantic and Syntactic Perspectives, Torino (Italia): Rosenberg and Sellier, 11-26.

Brea, M. 1994. A propósito del prefijo des-. B. Pallares, P. Peira y J. Sánchez Lobato (eds.), Homenaje a María Josefa Canellada, Madrid: Editorial Complutense, 111-124.

Cifuentes Honrubia, J. L. 1999. Sintaxis y semántica del movimiento. Aspectos de gramática cognitiva, Alicante: IAC Juan Gil-Albert.

Cifuentes Honrubia, J. L. 2006. Verbos denominales locales en español. E. De Miguel, A. Palacios, A. Serradilla (eds.), Estructuras léxicas y estructuras del léxico, Francfort del Meno: Peter Lang, 247-271.

Cifuentes Honrubia, J. L. 2011. Spanish deadjectival verbs and argument structure. J. L. Cifuentes y S. Rodríguez Rosique (eds.), Spanish Word Formation and Lexical Creation, Amsterdam / Philadelphia: John Benjamins, 65-105.

Clark, E. V. y Clark, H. H. 1979. When nouns surface as verbs. Language, 55, 767-811.

Costa, S. 2008. Negación y prefijación negativa. Algunos aspectos de la forma y la interpretación de las secuencias negativas, Montevideo: Universidad de la República.

Cruse, D. A. 1979. Reversives. Linguistics, 17, 957-966.

Cruse, D. A. 1986. Lexical Semantics, Cambridge: Cambridge University Press.

De Miguel, E. 1999. El aspecto léxico. I. Bosque y V. Demonte (dirs.), Gramática descriptiva de la lengua española, Madrid: Espasa-Calpe, 2977-3060.

Declerck, R. 1979. Aspect and the bounded / unbounded (telic / atelic) distinction. Linguistics, 17, 761-794.

Demonte, V. 1994. La semántica de los verbos de cambio. Cuadernos de Lingüística del I.U. Ortega y Gasset, 2, 55-82.

Demonte, V. y McNally, L. 2012. Introduction. L. McNally y V. Demonte (eds.), Telicity, 
Change and State. A Cross-categorial View of Event Structure, Oxford: Oxford University Press, 1-19.

Dowty, D. 1979. Word meaning and Montague Grammar, Reidel: Dordrecht.

Dowty, D. 1991. Thematic Proto-Roles and argument selections. Language, 67:3, 547-619.

Givón, T. 1978. Negation in Language: Pragmatics, Function, Ontology. P. Cole (ed.), Syntax and Semantics. Vol. 9: Pragmatics, 69-112.

Grossmann, M. 1994. Opposizioni direzionali e prefissazione. Analisi morfologica e semantica dei verbi egressivi prefissati con des- e es- in catalano, Padua: Unipress.

Hay, J., Kennedy, C. y Levin, B. 1999. Scalar structure underlies telicity in 'degree achievements'. T. Mathews y D. Strolovitch (eds.), SALT IX, CLC Publications, 127-144.

Horn, L. [1984] 1998. Toward a new Taxonomy for Pragmatic Inference: Q-based and Rbased Implicature. A. Kasher (ed.), Pragmatics. IV, Londres: Routledge, 383-418.

Horn, L. 1988. Morphology, pragmatics and the un-verb, ESCOL (5 ${ }^{\text {th }}$ Eastern State Conference on Linguistics), 1988, 210-233.

Horn, L. [1989] 2001. A Natural History of Negation, Stanford: CSLI Publications.

Horn, L. 2002. Uncovering the un-word: A study in lexical pragmatics. Sophia Linguistica, 49, 1-64.

Jeschull, L. 2007. The pragmatics of telicity and what children make of it. A. Belikova, L. Meroni, y M. Umeda (eds.), Proceedings of the 2nd Conference on Generative Approaches to Language Acquisition North America (GALANA), MA: Cascadilla Proceedings Project, 180-187.

Kearns, K. 2000. Semantics, Nueva York: St. Martin’s.

Kearns, K. 2007. Telic senses of deadjectival verbs. Lingua, 117, 26-66.

Kennedy, C. y McNally, L. 2005. Scale Structure, Degree Modification and the Semantics of Gradable Predicates. Language, 81:2, 345-381. 
Kennedy, C. 2012. The composition of incremental change. L. McNally y V. Demonte (eds.), Telicity, Change and State. A Cross-categorial View of Event Structure, Oxford: Oxford University Press, 103-121.

Koontz-Garboden, A. y Levin, B. 2005. The morphological typology of change of state event encoding. G. Booig, E. Guevara, A. Ralli, S. Sgroi y S. Scalise (eds.), Morphology and Linguistic Typology. On-line Proceedings of the Fourth Mediterranean Morphology Meeting (MMM4) Catania 21-23 September 2003, Universidad de Bolonia http://morbo.lingue.unibo.it/mmm/

Krifka, M. 1998. The origins of telicity. S. Rothstein (ed.), Events and Grammar, Dordrecht: Kluwer, 197-235.

Lavale Ortiz, R. M. 2007. Causatividad y verbos denominales. Estudios de Lingüística de la Universidad de Alicante (ELUA), 21, 171-207.

Levinson, S. 2000. Presumptive Meanings. The Theory of Generalized Conversational Implicature, Cambridge / Londres: The MIT Press.

Lieber, R. 2004. Morphology and Lexical Semantics, Cambridge: Cambridge University Press.

Lyons, J. 1977. Semantics, Cambridge: Cambridge University Press. 2 vols.

Marchand, H. [1973] 1974. Reversative, Ablative, and Privative Verbs in English, French, and German. D. Kastovsky (ed.), Studies in Syntax and Word-Formation. Selected Articles by Hans Marchand, Munich: Wilhelm Fink Verlag, 405-415.

Martín García, J. 2007. Las palabras prefijadas con des-. Boletín de la Real Academia Española, LXXXVII- CCXCV, 5-27.

Montero Curiel, M. L. 1999. La prefijación negativa en español, Cáceres: Universidad de Extremadura.

Olsen, M. B. 1994. The semantics and pragmatics of lexical aspect features. Studies in the Linguistic Sciencies, 24, 1/2, 361-375.

Paradis, C. 2001. Adjectives and boundedness. Cognitive Linguistics, 12, 47-64. 
Piñón, C. 1997. Achievements in an Event Semantics. A. Lawson (ed.), SALT VII, Nueva York: Cornell University, 276-293.

Pustejovsky, J. [1991] 2005. The syntax of event structure. I. Mani, J. Pustejovsky y R. Gaizauskas (eds.), The Language of Time: A Reader, Oxford: Oxford University Press, 33-60.

Rappaport Hovav, M. 2008. Lexicalized meaning and the internal temporal structure of events. S. Rothstein (ed.), Theoretical and Crosslinguistic Approaches to the Semantics of Aspect, Amsterdam / Philadelphia: John Benjamins, 13-42.

Rappaport Hovav, M. y Levin, B. 2010. Reflections on manner / result complementarity. M. Rappaport Hovav, E. Doron e I. Sichel (eds.), Lexical Semantics, Syntax and Event Structure, Oxford: Oxford University Press. 21-58.

Real Academia Española [R.A.E.]. 2001. Diccionario de la Lengua Española, Madrid: Espasa Calpe.

Real Academia Española [R.A.E.]. 2009. Nueva Gramática de la Lengua Española, Madrid: Espasa Calpe. Vol. I.

Real Academia Española [R.A.E.]. Banco de datos (CREA). Corpus de referencia del español actual. http://www.rae.es

Rifón, A. 1997. Pautas semánticas para la formación d verbos en español mediante sufijación, Santiago de Compostela: Universidad de Santiago de Compostela.

Rodríguez Rosique, S. 2008. Pragmática y gramática. Condicionales concesivas en español, Francfort del Meno: Peter Lang.

Rothstein, S. 2012. Another look at accomplishments and incrementality. L. McNally y V. Demonte (eds.), Telicity, Change and State. A Cross-categorial View of Event Structure, Oxford: Oxford University Press, 60-102.

Serrano Dolader, D. 1995. Las formaciones parasintéticas en español, Madrid: Arco.

Serrano Dolader, D. 1999. La derivación verbal y la parasíntesis. I. Bosque y V. Demonte (dirs.), Gramática descriptiva de la lengua española, Madrid: Espasa-Calpe, 4683-4755. 
Serrano Dolader, D. 2011. Base selection and prefixing: The prefix des-. J. L. Cifuentes Honrubia y S. Rodríguez Rosique (eds.), Spanish Word Formation and Lexical Creation, Amsterdam: John Benjamins, 255-281.

Talmy, L. 2000. Towards a Cognitive Semantics, Cambridge, Massachusetts: The MIT Press, Vol. 2.

Tenny, C. 1995. How motion verbs are special: The interaction of semantic and pragmatic information in aspectual verb meanings. Pragmatics and Cognition. 3 (1), 31-73.

Vañó-Cerdá, A. 1990. Las correspondencias del prefijo español des- con los afijos y adverbios alemanes (mi $\beta$-, ent-, zurück-, zer-, -los, los-, un-, etc.). Ibero-Romania, 31, 1-27.

Varela Ortega, S. y Martín García, J. 1999. La prefijación. I. Bosque y V. Demonte (dirs.), Gramática descriptiva de la lengua española, Madrid: Espasa-Calpe, 4993-5040.

Vendler, Z. 1957. Verbs and Times. The Philosophical Review, 66, 2, 143-160.

Recibido: 16 abril 2013

Aceptado: 23 abril 2013

Revisado: 29 mayo 2013

Publicado: 31 mayo 2013 